\title{
Influência da precipitação da fase $\Omega$ na dureza das ligas - Ti-10Mo-20Nb e Ti-12Mo-13Nb
}

\section{Influence of $\Omega$ precipitation in the hardness of Ti-10Mo-20Nb and Ti12Mo- $13 \mathrm{Nb}$ Alloys}

Artigo

Original

\author{
Laiz Silva Mansur ${ }^{1}$ \\ Juliana Torres ${ }^{2}$ \\ Luiz Henrique De Almeida ${ }^{3}$ \\ Carlos Angelo Nunes ${ }^{4}$ \\ Iranildes dos Santos ${ }^{5}$ \\ Sinara Borborema Gabriel ${ }^{6}$
}

Original

Paper

Recebido em
05/2011

Aprovado em 03/2012

\section{Palavras-chave: \\ Ligas de Titânio \\ Envelhecimento}

Dureza

Biomateriais

\begin{abstract}
Resumo:
Ligas de Ti, do tipo $\beta$ tem sido desenvolvidas para aplicação biomédica. As vantagens destas ligas incluem seu baixo módulo de elasticidade, boa compatibilidade mecânica e, além disso, as variáveis de processamento podem ser controladas para obter propriedades desejadas. As ligas de Ti- $\beta$, na condição metaestável, podem ser fortemente endurecidas por precipitação de fases após envelhecimento. Várias microestruturas podem ser obtidas por diferentes tratamentos de envelhecimento os quais influenciam várias propriedades das ligas Diante do contexto, o objetivo deste trabalho foi estudar a influência da precipitação da fase $\omega$ na dureza das ligas Ti-10Mo-20Nb e Ti-12Mo-13Nb. O material nas diferentes condições foi caracterizado por difração de raios $\mathrm{X}$ e medida de microdureza. Os resultados mostraram que a precipitação da fase $\omega$ durante o envelhecimento acarretou no aumento da dureza das ligas Ti$10 \mathrm{Mo}-20 \mathrm{Nb}$ e Ti-12Mo-13Nb. Observou-se uma tendência da liga Ti$12 \mathrm{Mo}-13 \mathrm{Nb}$ apresentar os maiores valores de dureza.
\end{abstract}

\begin{abstract}
$\beta$-type Ti alloys, has been developed for biomedical application. The advantages of these alloys include its low modulus and good mechanical compatibility; in addition, the processing variables can be controlled to obtain desired properties. The $\beta$-Ti alloys in the metastable condition can be significantly hardened by precipitation of the phases after aging. Various microstructures can be obtained by different aging treatments which influence various properties of the alloys. At this context, the objective was to study the influence of $\omega$ phase precipitation on the hardness of Ti-10Mo-20Nb and Ti-13Nb12 Mo alloys. The material in different conditions was characterized by $X$-ray diffraction and microhardness measurement. The results showed than the precipitation of $\omega$ phase during aging resulted in hardness increase of Ti-10Mo-20Nb and Ti-13Nb-12Mo alloys. It was observed a tendency of Ti-13Nb-12Mo show higher hardness values.
\end{abstract}

Key words:

Titanium alloys

Aging

Hardness

Biomaterials

\footnotetext{
1.Acadêmico do curso de Engenharia de Produção do Centro Universitário de Volta Redonda - UniFOA

2.Acadêmico do curso de Engenharia de Produção do Centro Universitário de Volta Redonda - UniFOA

3.Doutor em Engenharia Metalúrgica e de Materiais pela Universidade Federal do Rio de Janeiro - COPPE/UFRJ - Rio de Janeiro

4.Doutor em Engenharia Mecânica pela Universidade Estadual de Campinas - UNICAMP-Campinas

5.Doutora em Engenharia Metalúrgica e de Materiais pela Universidade Federal do Rio de Janeiro - COPPE/UFRJ - Rio de Janeiro
} 


\section{Introdução}

Titânio e suas ligas são amplamente usados como implantes ortopédicos por apresentarem resistência à corrosão, biocompatibilidade, maior resistência e menor módulo de elasticidade que outros biomateriais metálicos tais como aço inoxidável e as ligas a base de Co-Cr [ZHOU et al., 2005].

Estudos recentes têm visado o desenvolvimento de novas ligas de Ti, do tipo $\beta$, compostas de elementos não tóxicos, como $\mathrm{Nb}$, $\mathrm{Ta}, \mathrm{Mo}, \mathrm{Zr}$ e Sn. As vantagens dessas ligas incluem seu baixo módulo de elasticidade, boa compatibilidade mecânica [MATSUMOTO et al., 2008] e, além disso, as variáveis de processamento podem ser controladas para obter propriedades desejadas [OLIVEIRA et al., 2007].

A metalurgia do Ti e suas ligas têm sido intensivamente estudadas nos últimos 50 anos [HENRIQUES et al., 2001]. Várias microestruturas podem ser obtidas por diferentes tratamentos de envelhecimento os quais influenciam várias propriedades das ligas e, portanto, um satisfatório tratamento de envelhecimento poderá resultar num melhor balanço entre baixo módulo de elasticidade e alta resistência [ZHOU et al., 2004].

As ligas de Ti- $\beta$ são classificadas como estáveis e metaestáveis, sendo que as ligas na condição metaestável são tratáveis e fortemente endurecidas por precipitação de fases após envelhecimento [PETERS et al., 2005]. $\mathrm{O}$ tipo de tratamento térmico tem uma função importante na determinação das propriedades dessas ligas. O efeito deste depende da composição química da liga, tempo e temperatura do tratamento térmico e trabalho a frio. Esses fatores determinam as transformações de fase [ANKENS \& EAGLE, 1983].

As fases que podem precipitar nas ligas de Ti- $\beta$ metaestável são: $\alpha$ que é uma fase de equilíbrio que se forma quando as ligas de $\mathrm{Ti}$ são tratadas abaixo da temperatura de transição $\beta$; fase $\omega$ que se forma em ligas de Ti- $\beta$ metaestável pobres em estabilizador $\beta$, podendo se formar atérmicamente ou isotermicamente, sendo que neste último se forma em temperaturas de $200-500{ }^{\circ} \mathrm{C}$; fase $\beta$ ' é uma fase metaestável pobre em estabilizador $\beta$ que se forma em ligas de Ti- $\beta$ metaestáveis ricas em estabilizador e onde não há precipitação de fase $\omega$ e, fase martensítica ( $\alpha$ ") induzida por tensão que pode se formar em ligas de Ti- $\beta$ pobres em estabilizador [ANKENS \& EAGLE, 1983].

Estudos realizados por Gabriel et al. [GABRIEL, 2008; GABRIEL et al., 2008] sobre o processamento das ligas Ti-10Mo-20Nb e Ti-12Mo-13Nb do tipo $\beta$ mostraram que estas ligas têm grande potencial para serem utilizadas como implantes ortopédicos. Com o intuito de entender melhor a precipitação de fases nestas ligas o objetivo deste trabalho, foi estudar a precipitação da fase $\omega$ e sua influência na dureza destas ligas.

\section{Procedimento Experimental}

As ligas Ti-10Mo-20Nb e Ti-12Mo$13 \mathrm{Nb}$ foram forjadas a frio até 74 e $80 \%$ de redução em área, respectivamente. Em seguida, passaram por um envelhecimento na temperatura de $300{ }^{\circ} \mathrm{C}$ por $10 \mathrm{~min}, 45 \mathrm{~min}$ e $3 \mathrm{~h}$.

As análises de fases das ligas nas diferentes condições na forma polida foram realizadas por difração de raios-X (DRX) usando uma Shimadzu modelo DRX 6000 nas seguintes condições: radiação $\mathrm{CuK} \alpha(\lambda$ $=1,5418 \AA$ ) com monocromador de grafite, tensão de $40 \mathrm{kV}$, corrente de $30 \mathrm{~mA}$, varredura (20) de 30 a 90 graus, passo angular de $0,05^{\circ} \mathrm{e}$ tempo de contagem de $5 \mathrm{~s}$ por ponto. As fases foram identificadas através da comparação com difratograma simulado. As simulações foram realizadas através do programa Powdercell [KRAUS \& NOLZE, 1996] inserindo dados das fases $\beta$ e $\omega$ [VILLARS \& CALVERT, 1991], como grupo espacial, parâmetros de rede e posições atômicas.

A microdureza da liga nas diferentes condições (polidas) foi medida utilizando um equipamento Micromet 2004, Buehler, com uma carga de 200 gf durante 30 s. Os valores de microdureza representam a média de 5 medidas.

\section{Resultados e discussão}

\subsection{Dureza}

Os valores médios (com os desvios-padrão) de microdureza Vickers para as 
ligas Ti-10Mo-20Nb e Ti-12Mo-13Nb, na condição forjada e envelhecidas após forjamento na temperatura de $300^{\circ} \mathrm{C}$ por 10 min, 45 min e $3 \mathrm{~h}$, seguido de resfriamento em água são mostrados na Figura 1. As curvas dos materiais envelhecidos mostram um comportamento típico de endurecimento por precipitação. bservou-se que, enquanto houve um aumento da dureza na liga Ti-12Mo-
$13 \mathrm{Nb}$ com 10 min de envelhecimento, o valor da dureza da Ti-10Mo-20Nb permaneceu constante em relação a condição forjada. Os maiores valores de dureza foram obtidos no envelhecimento por $3 \mathrm{~h}$.

Observou-se uma tendência da liga Ti$12 \mathrm{Mo}-13 \mathrm{Nb}$ apresentar os maiores valores de dureza o que pode estar relacionado com a maior precipitação de fase.

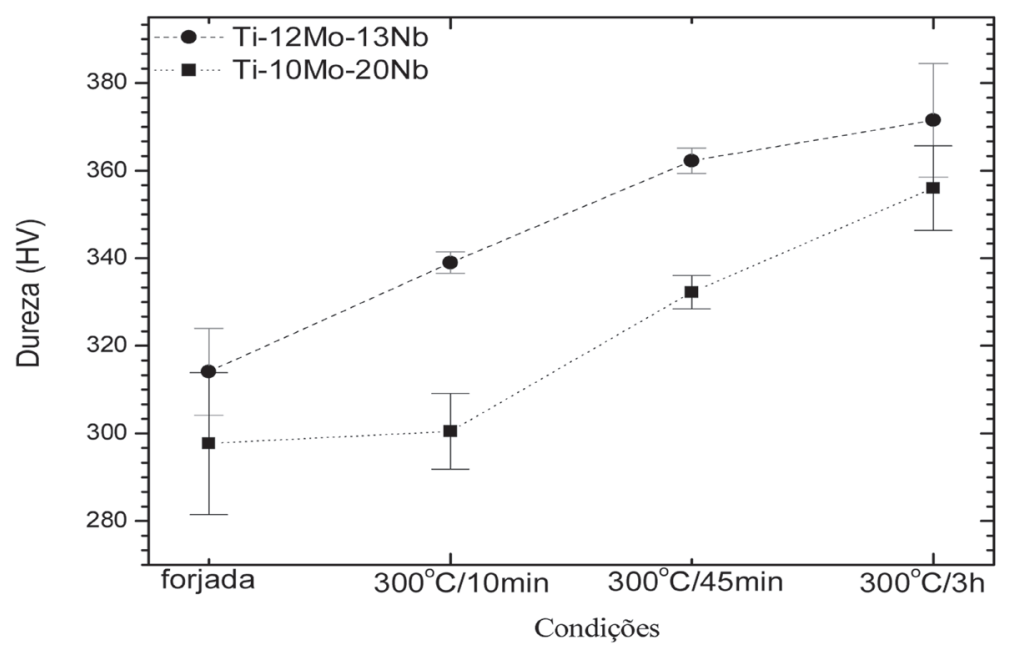

Figura 1 - Curvas de microdureza das ligas Ti-10Mo-20Nb e Ti-12Mo-13Nb nas diferentes condições de processamento.

\subsection{Difração de raios $X$}

A Figura 2 mostra os padrões de DRX da liga Ti-10Mo-20Nb (a) na condição forjada, (b) após envelhecimento a $300^{\circ} \mathrm{C} / 10 \mathrm{~min}$, (c) após envelhecimento a $300^{\circ} \mathrm{C} / 45 \mathrm{~min}$ e (d) após envelhecimento a $300^{\circ} \mathrm{C} / 3 \mathrm{~h}$. No difratograma da amostra na condição forjada só foi possível identificar a presença da fase $\beta$, estrutura cúbica de corpo centrado (ccc). Observou-se a presença de fase $\omega$ na matriz $\beta$ no envelhecimento a partir de $10 \mathrm{~min}$.

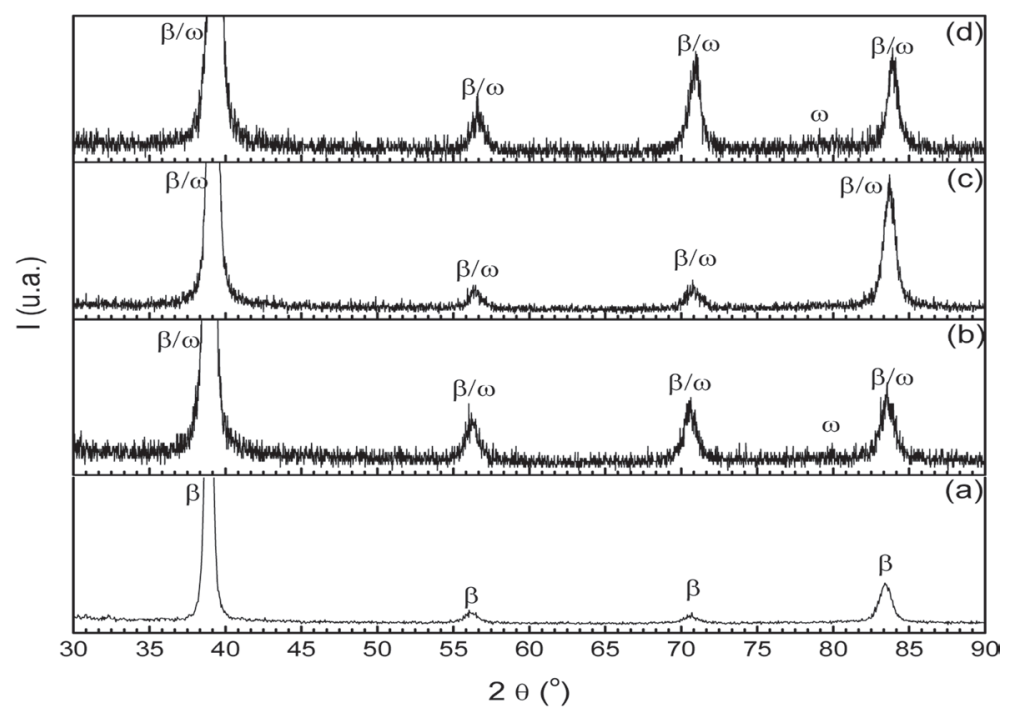

Figura 2 - Difratogramas da liga Ti-10Mo-20Nb (a) na condição forjada(b) após envelhecimento a $300^{\circ} \mathrm{C} / 10 \mathrm{~min}$, (c) após envelhecimento a $300^{\circ} \mathrm{C} / 45 \mathrm{~min}$ e (d) após envelhecimento a $300^{\circ} \mathrm{C} / 3 \mathrm{~h}$. 
A Figura 3 mostra os difratogramas da liga Ti-12Mo-13 $\mathrm{Nb}$ (a) na condição forjada, (b) após envelhecimento a $300^{\circ} \mathrm{C} / 10 \mathrm{~min}$, (c) após envelhecimento a $300^{\circ} \mathrm{C} / 45 \mathrm{~min}$ e (d) após envelhecimento a $300^{\circ} \mathrm{C} / 3 \mathrm{~h}$. No difratograma da amostra na condição forjada só foi possível identificar a presença da fase $\beta$, estrutura cúbica de corpo centrado (ccc). Observou-se a presença de fase $\omega$ na matriz $\beta$ no envelhecimento a partir de $10 \mathrm{~min}$ e as reflexões referentes a fase $\omega$ se tornaram mais intensas com o aumento do tempo do envelhecimento.
Comparando os difratogramas das ligas Ti$10 \mathrm{Mo}-20 \mathrm{Nb}$ e Ti-12Mo-13Nb, verificou-se que houve uma maior precipitação de fase $\omega$ na liga Ti-12Mo-13Nb durante o envelhecimento que está de acordo com os resultados de dureza para estas ligas, onde observa-se que os maiores valores de dureza foram obtidos para a liga Ti-12Mo-13Nb (mais rica em Mo), sugerindo que este elemento torna a liga mais propensa a formação de fase $\omega$. Esse aumento de dureza com a precipitação da fase $\omega$ está de acordo com a literatura que mostra que esta fase acarreta no aumento da dureza das ligas de Ti [MATSUMOTO et al., 2007].

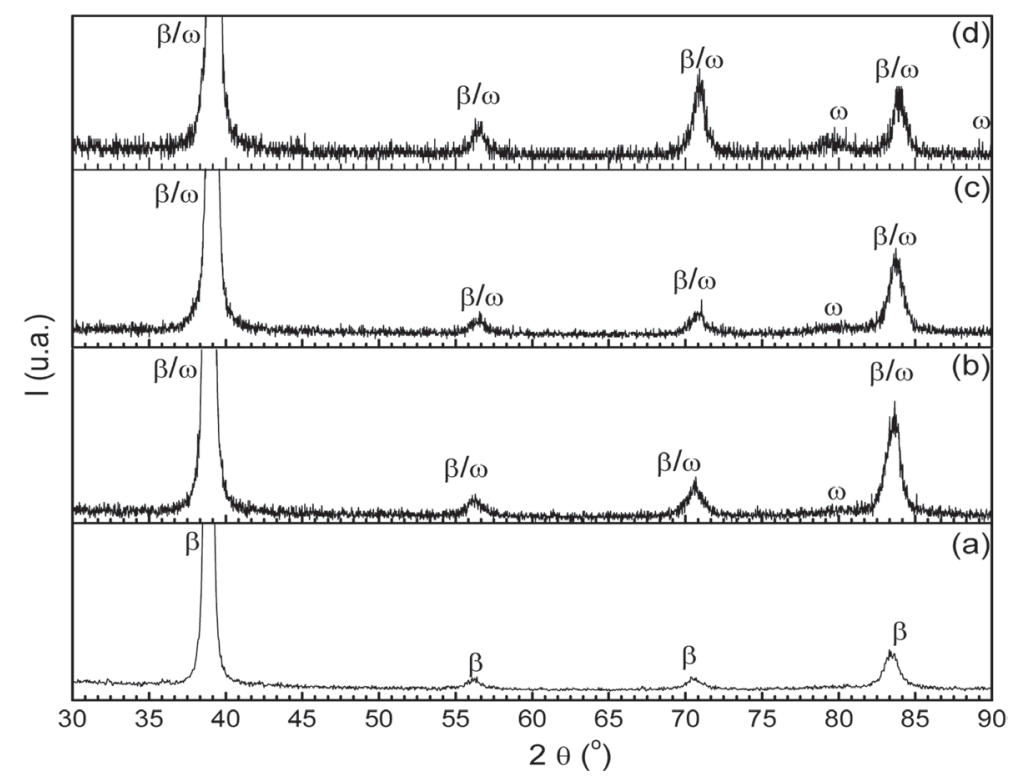

\section{Conclusão}

Conclui-se que a precipitação da fase $\omega$, durante o envelhecimento, acarretou no aumento da dureza das ligas Ti-10Mo-20Nb e Ti-12Mo-13Nb. Observou-se uma tendência da liga Ti-12Mo-13Nb apresentar os maiores valores de dureza.

Verificou-se que houve uma maior precipitação de fase $\omega$ na liga Ti-12Mo-13Nb durante o envelhecimento que está de acordo com os resultados de dureza para essas ligas, onde observa-se que os maiores valores de dureza foram obtidos para a liga Ti-12Mo-13Nb (mais rica em Mo), sugerindo que este elemento torna a liga mais propensa a formação de fase $\omega$.

\section{Agradecimentos}

Os autores agradecem ao Departamento de Engenharia de Materiais da Escola de Engenharia de Lorena (EEL/USP), a Universidade Federal do Rio de Janeiro (COPPE/UFRJ) e ao NUPE/UniFOA pelo desenvolvimento deste trabalho. 


\section{Referências Bibliográficas}

1. ANKEN, S., SEAGLE, S. R., Heat Treatment of Metastable Beta Titanium Alloys". In: Proceedings of a symposium sponsored by the Titanium Committee of AIME, held at the Annual Meeting of The Metallurgical Society, p. 107126, Atlanta, Georgia, March, 1983.

2. GABRIEL, Sinara Borborema. Processamento e caracterização de ligas Ti-Mo-Nb para aplicações biomédicas, 2008, Tese de Doutorado, COPPE/UFRJ, Rio de Janeiro, 2008.

3. GABRIEL, S. B., NUNES C. A., DILLE, J., SOARES, G. A. Microstructural evolution and Mechanical properties of aged $\mathrm{Ti}-12 \mathrm{Mo}-13 \mathrm{Nb}$ alloy. In Congresso Brasileiro de Engenharia e Ciências dos Materiais, Porto de Galinhas, 2008.

4. HENRIQUES, V. A. R., BELLINATI, C. E., SILVA, C. R. M., Production of Ti$6 \% \mathrm{Al}-7 \% \mathrm{Nb}$ alloy by powder metallurgy (P/M)", Journal of Materials Processing Technology, v. 118, p. 212-215, 2001.

5. KRaUS W. AND NOLZE, G. J. Appl. Cryst., v.29, 301-303, 1996.

6. MATSUMOTO, H., WATANABE, S., HANADA, S., Microstructures and mechanical properties of metastable $\beta$ TiNbSn alloys cold rolled and heat treated, Journal of Alloys Compounds, v.439, pp. 146-155, 2007.
7. MATSUMOTO, H., CHIBA, A., HANADA, S., Anisotropy of Young's modulus and tensile properties in cold rolled $\alpha^{\prime}$ martensite Ti-V-Sn alloys, Materials Science and Engineering A, v. 486, p. 503-510, 2008.

8. OLIVEIRA,N.T.C.,ALEIXO,G.,CARAM, R., et al., Development of Ti-Mo alloys for biomedical applications: Microstructure and electrochemical characterization, Materials Science and Engineering A, v. 452-453, p. 727-731, 2007.

9. PETERS, M., HEMPTENMACHER, J., KUMPFERT, J. et al, Structure and Properties of Titanium and Titanium Alloys". In: Leyens, C., Peters, M. (eds), Titanium and Titanium Alloys, 2 ed., chapter 1, Cologne, Germany, WILEY VCH GmbH \&Co.KGaA, 2005.

10. VILLARS, P.; CALVERT, L.D. Pearson's Handbook of Crystallographic Data for Intermetallic phases, $2^{\circ}$ ed., ASM International, Materials Park, four v., 1991.

11. ZHOU, Y. L., NIINOMI, M., AKAHORI, T., et al., Corrosion resistance and biocompatibility of Ti-Ta alloys for biomedical applications, Materials Science and Engineering A, v. 398, p. 28-36, 2005.

12. ZHOU, Y. L., NIINOMI, M., AKAHORI, T., Decomposition of martensite $\alpha$ " during aging treatments and resulting mechanical properties of Ti-Ta alloys, Materials Science and Engineering A, v. 384, p. 92-101, 2004.
Endereço para Correspondência:

Sinara Borborema Gabriel

sinarab@msn.com

Centro Universitário de Volta Redonda - UniFOA

Campus Olezio Galotti

Av. Paulo Erlei Alves Abrantes, $n^{\circ} 1325$

Três Poços - Volta Redonda - RJ

CEP: $27240-000$

Informações bibliográficas:

Conforme a NBR 6023:2002 da Associação Brasileira de Normas Técnicas (ABNT), este texto científico publicado em periódico eletrônico deve ser citado da seguinte forma: MANSUR, Laiz Silva; TORRES, Juliana; ALMEIDA, Luiz Henrique De; NUNES, Carlos Angelo; SANTOS, Iranildes dos; GABRIEL, Sinara Borborema. Influência da unifoa.edu.br/cadernos/edicao/18/27.pdf $>$ 